\title{
Association of CACNG6 polymorphisms with aspirin-intolerance asthmatics in a Korean population
}

\author{
Jin Sol Lee ${ }^{1}$, Jeong-Hyun Kim¹, Joon Seol Bae', Jason Yongha Kim¹, Tae Joon Park', Charisse Flerida Pasaje', \\ Byung-Lae Park ${ }^{2}$, Hyun Sub Cheong ${ }^{2}$, Soo-Taek Uh³ ${ }^{3}$ Jong-Sook Park ${ }^{4}$, An-Soo Jang ${ }^{4}$, Mi-Kyeong Kim², \\ Inseon S Choi ${ }^{6}$, Choon-Sik Park ${ }^{3 *}$, Hyoung Doo Shin ${ }^{1,2^{*}}$
}

\begin{abstract}
Background: Aspirin-intolerant asthma (AIA) occurs in the lower and upper airways through excessive production of leukotrienes upon administration of non-steroidal anti-inflammatory drugs (NSAIDs). One of the three symptoms of AIA is nasal polyposis, a chronic inflammatory disease that is related to the function of calcium ion in recruitment of immune cells during airway inflammation. It has been implicated that bronchodilation in the airway is related to $\mathrm{Ca}(2+)$ regulation. The calcium channel, voltage-dependent, gamma subunit 6 (CACNG6) gene encodes a protein that stabilizes the calcium channel.
\end{abstract}

Methods: To study the associations between AIA and polymorphisms in CACNG6 gene, eight variants were genotyped in 102 AIA cases and 429 aspirin-tolerant asthma (ATA) controls. Logistic analyses were used to evaluate the associations of CACNG6 polymorphisms with AIA.

Results: Statistical analyses revealed that a single nucleotide polymorphism (SNP; rs 192808C $>$ T; $P=0.0004$, $P^{\text {corr }}=$ $0.0029, \mathrm{OR}=2.88$ in co-dominant model; $P=0.0005, P^{\text {corr }}=0.0036, \mathrm{OR}=2.99$ in dominant model) in intron and a haplotype unique to this variant (CACNG6_BL1_ht6; $P=0.003, P^{\text {corr }}=0.02, \mathrm{OR}=2.57$ in co-dominant model, $P=$ $0.001, P^{c o r r}=0.0087, \mathrm{OR}=2.81$ in dominant model) were significantly associated with the risk of AIA.

Conclusions: Our results suggest that the CACNG6 variants might be associated with the risk of AIA in a Korean population.

\section{Background}

Aspirin-intolerant asthma (AIA), which was first described in 1922, is a unique clinical syndrome associated with acute bronchconstriction upon administration of aspirin and other non-steroidal anti-inflammatory drugs [1]. Although considered as one of the most widely used medication, aspirin intake also incurs various side effects including manifested gastrointestinal ulcer, stomach bleeding and tinnitus especially in higher doses. Although the side effects caused by aspirin are

\footnotetext{
* Correspondence: schalr@schbc.ac.kr; hdshin@sogang.ac.kr

'Department of Life Science, Sogang University, Seoul 121-742, Republic of Korea

${ }^{3}$ Division of Allergy and Respiratory Medicine, Soonchunhyang University Seoul Hospital, Seoul 140-743, Republic of Korea

Full list of author information is available at the end of the article
}

not common, these effects have been reported in about $10 \%$ of adult asthmatics.

The triad of AIA symptoms includes aspirin sensitivity, bronchial asthma and chronic rhinosinusitis with nasal polyposis [2-4]. Nasal polyposis is a chronic inflammatory disease of the upper respiratory tract that affects around two thirds of patients with AIA. The polyps are filled with mast cells and eosinophils due to high levels of the proinflammatory cysteinyl leukotrienes (cysLTs) [5]. After mast cells are activated, it releases a variety of paracrine signals that target tissues such as bronchi and vasculature to recruit other immune cells at the inflammatory site. Among the remarkable signaling molecules released by mast cells, the cysLTs that includes leukotriene C(4) (LTC4), LTD4, and LTE4 are well known [6,7]. The LTC4 is secreted from mast cells
C Biomed Central 
following $\mathrm{Ca}(2+)$ influx through store-operated calcium release-activated calcium (CRAC) channels [7-10]. It has also been reported that proteinase activated receptor-2 (PAR2), as a novel emerging pharmacological target in airway diseases, is expressed in the airway epithelium and its activation may be involved in the protective role of preserving bronchial functionality [11,12]. In line with the fact that the airway smooth muscle cell contraction is regulated by changes in intracellular $\mathrm{Ca}(2+)$ concentration, a previous molecular report has revealed a relationship between this $\mathrm{Ca}(2+)$ regulation and bronchodilation in the airway [13]. More recently, novel actions of NSAIDs on vascular ion channels including L-type calcium channel has also been suggested [14].

The L-type calcium channels are composed of five subunits. The CACNG6 gene encodes one of these subunits, specifically a gamma subunit protein which was first identified in muscle cells [15]. Recent studies have revealed negative relations of $C A C N G 6$ gene expression to chronic obstructive pulmonary disease, responses of the human airway epithelium following injury and \% parenchyma in lung tissues $[16,17]$. The CACNG6 is an integral membrane protein that stabilizes the calcium channel during its inactive state [18]. Since the role of azelastine in asthma treatment is achieved by blocking the L-type calcium channel thus, preventing $\mathrm{Ca}(2+)$ current [19], the CACNG6 may, therefore, be of interest in the study of asthma treatment. Moreover, abnormal $\mathrm{Ca}(2+)$ influx may induce leukotriene overproduction from mast cells, leading to recruitment of other immune cells, such as eosinophils, resulting in vasospasm by eosinophilic infiltration of the coronary artery wall and cardiac contractility $[8,20]$. Although many signaling pathways have been known to underlie the reversible contraction of airway smooth muscle, ionic mechanisms related to $\mathrm{Ca}(2+)$ and the calcium channels in the airways are still poorly understood [21]. In this connection, we explored whether CACNG6 single nucleotide polymorphisms (SNPs) are associated with AIA.

\section{Methods}

\section{Study Subjects}

Subjects in this study were recruited from the Asthma Genome Research Center comprising hospitals of Soonchunhyang, Chunnam, Chungbuk, Seoul National and Chung-Ang Universities in Korea. All subjects provided written informed consents and the study protocols were approved by the Institutional Review Board of each hospital. Diagnosis of AIA was performed according to a modified method as previously described [22]. We also performed aspirin challenge in subjects with a history of aspirin hypersensitivity, presence of urticaria, nasal polyps and sinusitis. The AIA case group included patients with $20 \%$ or greater decreases in $\mathrm{FEV}_{1}$ or $15 \%$ to $19 \%$ decreases in $\mathrm{FEV}_{1}$ with naso-ocular or cutaneous reactions, whereas subjects showing a rate of $\mathrm{FEV}_{1}$ decline less than $15 \%$ without extrabronchial, nasal or skin symptoms were included in ATA group.

\section{SNP selection and genotyping}

We selected 8 common polymorphic SNPs from the International HapMap Project phase 1\&2 http://hapmap. ncbi.nlm.nih.gov/ and National Center for Biotechnology Information (build 36) based on the minor allele frequency (>0.05) and LD status. Among three SNPs $(r s 3810244, r s 158194, r s 4806481)$ in LD $\left(\left|\mathrm{D}^{\prime}\right|>0.7\right)$, the most common SNP (rs4806481) was selected for genotyping. For examination of AIA risk association, we genotyped 8 common SNPs in the CACNG6 gene. Genotyping was carried out with 20 ng of genomic DNA by TaqMan assay in the ABI prism $7900 \mathrm{HT}$ sequence detection system software version 2.3 (Applied Biosystems, CA, USA) in 102 AIA cases and 429 ATA controls with the assessment of data quality by duplicate DNAs $(\mathrm{n}=10)$. A total of 8 polymorphisms on CACNG6 were successfully genotyped.

\section{Statistics}

We calculated linkage disequilibrium to all pairs of biallelic loci using Lewontin's D' (|D'|) [23] and $r^{2}$. PHASE algorithm (ver. 2.0) developed by Stephens et al. was used for inferring haplotypes [24]. Associations of genotypes and haplotypes in the CACNG6 gene with AIA were calculated using logistic analyses adjusted for age, gender, smoking status, atopy and body mass index as covariates. We also performed linear regression analysis to determine the differences in the rates of decline in $\mathrm{FEV}_{1}$ following aspirin challenge among the genotypes and haplotypes. The data were adjusted, managed and analyzed using SAS version 9.1 (SAS Inc., Cary, NC). The effective number of independent marker loci (7.28) was calculated for multiple testing corrections using SNPSpD software http://genepi. qimr.edu.au/general/daleN/SNPSpD/, which is based on the spectral decomposition $(\mathrm{SpD})$ of matrices of pair-wise LD between SNPs.

\section{Results}

In this study, the clinical characteristics of the study subjects showed that the fall rate by aspirin provocation in AIA patients was significantly higher than that of ATA controls $(33.59 \pm 13.42 \%$ vs. $3.54 \pm 4.85 \%$, respectively; $P<0.0001)$. This was in contrast to the current smoker ratio and mean age which was higher in ATA controls than those of AIA patients $(21.36 \%$ vs. $30.07 \%$ for smoking status and 42.76 year vs. 47.30 year for mean age, respectively). Body mass index (BMI) and PC20 methacholine test also showed significant signals $(P<0.01)$. In addition, AIA patients were more sensitive 
Table 1 Clinical profiles of aspirin intolerance asthma and control subjects

\begin{tabular}{|c|c|c|}
\hline Clinical profile & AIA & ATA \\
\hline Number of subjects (n) & 102 & 429 \\
\hline Age [year, mean (range)] & $\begin{array}{l}42.76(18.66- \\
72.73)^{*}\end{array}$ & $\begin{array}{l}47.30(15.40- \\
77.88)\end{array}$ \\
\hline Sex ( $n$, male/female) & $37 / 65$ & $147 / 282$ \\
\hline $\begin{array}{l}\text { Smoker (current smoker/exsmoker) } \\
(\%)\end{array}$ & $\begin{array}{l}21.36(13.59 / \\
7.77)^{*}\end{array}$ & $\begin{array}{l}30.07(12.35 / \\
17.72)\end{array}$ \\
\hline Height $[\mathrm{cm}$, mean (range)] & $161.70 \pm 9.91$ & $160.42 \pm 8.39$ \\
\hline Weight (kg) & $61.64 \pm 10.39$ & $63.40 \pm 10.97$ \\
\hline Body mass index $\left(\mathrm{kg} / \mathrm{m}^{2}\right)$ & $23.56 \pm 3.37^{*}$ & $24.58 \pm 3.39$ \\
\hline $\begin{array}{l}\% \text { decline of } \mathrm{FEV}_{1} \text { by aspirin } \\
\text { provocation }\end{array}$ & $33.59 \pm 13.42^{* *}$ & $3.54 \pm 4.85$ \\
\hline Blood eosinophil (\%) & $6.65 \pm 5.78$ & $6.03 \pm 5.92$ \\
\hline $\mathrm{FEV}_{1}$ (\% predicted) & $85.10 \pm 16.41^{*}$ & $91.66 \pm 16.87$ \\
\hline PC20 methacholine $(\mathrm{mg} / \mathrm{ml})$ & $4.26 \pm 7.62^{*}$ & $6.91 \pm 8.90$ \\
\hline Total lgE (IU/ml) & $415.74 \pm 714.70$ & $361.00 \pm 607.56$ \\
\hline Positive rate of skin test (\%) & 48.04 & 57.81 \\
\hline
\end{tabular}

Age indicates a first medical examination. AIA, aspirin-intolerant asthma; ATA, aspirin-tolerant asthma.

${ }^{*} P<0.05 ;{ }^{*} P<0.0001$ compared to ATA control.

to methacholine than ATA patients $(4.26 \mathrm{mg} / \mathrm{ml}$ vs. 6.91 $\mathrm{mg} / \mathrm{ml}$ ). Analyses on other factors in subject's clinical characteristics showed no significant differences between AIA and ATA groups. The clinical characteristics of the study subjects are summarized in Table 1 .

To investigate the association of CACNG6 polymorphisms with AIA, 8 SNPs ( $r 251850, r s 4806481, r s 158196$, rs158199, rs192808, rs450227, rs2291068, rs459247) in the CACNG6 gene were genotyped in a total of 531 study subjects including 102 AIA patients as a case group and 429 ATA patients as controls. The genotype distributions of all loci were in Hardy-Weinberg equilibrium (Table 2, $P>0.05$ ). Genetic map and location of CACNG6 polymorphisms are shown in Fig. 1A. Logistic analysis was used to analyze whether the genetic polymorphisms were associated with AIA. Interestingly, analysis revealed that a SNP in intron $(r s 192808 C>T$; OR $=2.88,95 \% \mathrm{CI}=1.60-5.17, P=0.0004, P^{\text {corr }}=0.0029$ in co-dominant model), which was more frequent in AIA patients than in ATA controls (0.103 in AIA patients and 0.045 in ATA patients) (Table 2), was significantly associated with the risk of AIA even after multiple testing corrections. In additional analysis using effective models, this allelic variant also showed a susceptibility to AIA in dominant model $(\mathrm{OR}=2.99,95 \% \mathrm{CI}=1.62$ 5.54, $\left.P=0.0005, P^{\text {corr }}=0.0036\right)$.

Based on the 8 polymorphisms, haplotypes were calculated using PHASE software resulting in two haplotype blocks (Fig. 1B and 1C). Although a different pattern in Block 1 between AIA and ATA was observed (Fig. 1D), the haplotypes which were composed of five SNPs were used for the association analysis because they were estimated as common haplotypes derived from a large number of subjects in the Korean population. Among the haplotypes, CACNG6_BL1_ht6, the only one containing the rare $\mathrm{T}$ variant of $r s 192808$, also showed significant association with risk of AIA $\left(P=0.003, P^{\text {corr }}=\right.$ 0.02 in co-dominant model; $P=0.001, P^{\text {corr }}=0.0087$ in dominant model), showing a similar pattern with the association of $r s 192808$ SNP (Table 2). In the case of CACNG6_BL1_ht6 haplotype, the frequency of AIA patients was higher at about two-fold than that of ATA controls (0.093 and 0.045, respectively).

Next, we performed regression analysis for the association between decline of $\mathrm{FEV}_{1}$ by aspirin provocation and CACNG6 polymorphisms. As summarized in Table 3 , among all the variants and haplotypes, SNP $r s 192808$ ( $P=0.006$ in co-dominant model and $P=0.01$ in dominant model) and haplotype CACNG6_BL1_ht6 $(P=0.03$ in co-dominant model and $P=0.02$ in dominant model) showed the most significant signal. In addition, in the second LD block, haplotype CACNG6_BL2_ht2 showed an association with the decline of $\mathrm{FEV}_{1}$ by aspirin provocation in the dominant model $(P=0.03)$. However, in further regression analysis using only AIA cases, no significant associations were detected (Additional file 1, Table S1), indicating that rs192808 and haplotypes of CACNG6 could not directly affect the aspirin-induced decline of $\mathrm{FEV}_{1}$ in AIA patients.

\section{Discussion}

Although over-production of pro-inflammatory cysLTs such as LTC4, LTD4 and LTE4 has been considered as a main cause of aspirin hypersensitivity in asthma [25], recent studies have suggested that other genes may be related to AIA $[21,26,27]$. On the other hand, previous studies have reported that concentration of calcium ion is related with leukotriene secretion [28] and that CACNG6 gene is negatively expressed in chronic obstructive pulmonary disease and human airway epithelium following injury $[16,17]$. Considering these facts, we hypothesized that the modulation of calcium concentration could play an important role in abnormal signaling pathways in the airways and that the CACNG6 gene, as a subunit of L-type calcium channels, and its polymorphisms might be associated with risk of AIA.

Although the minor $\mathrm{T}$ allele of $r s 192808$ and its unique haplotype (CACNG6_BL1_ht6) showed significant association signals with decline of $\mathrm{FEV}_{1}$ (Table 3), allelic dose effect revealed no consistent results of decline of $\mathrm{FEV}_{1}$ between rs192808 and CACNG6_BL1_ht6 due to the small number of rare alleles $(\mathrm{R} / \mathrm{R})$, therefore further replication studies are needed for a correct association. Among polymorphisms in the CACNG6 gene, the $r s 192808$ polymorphism in intronic region showed a highly significant signal. It has also been 


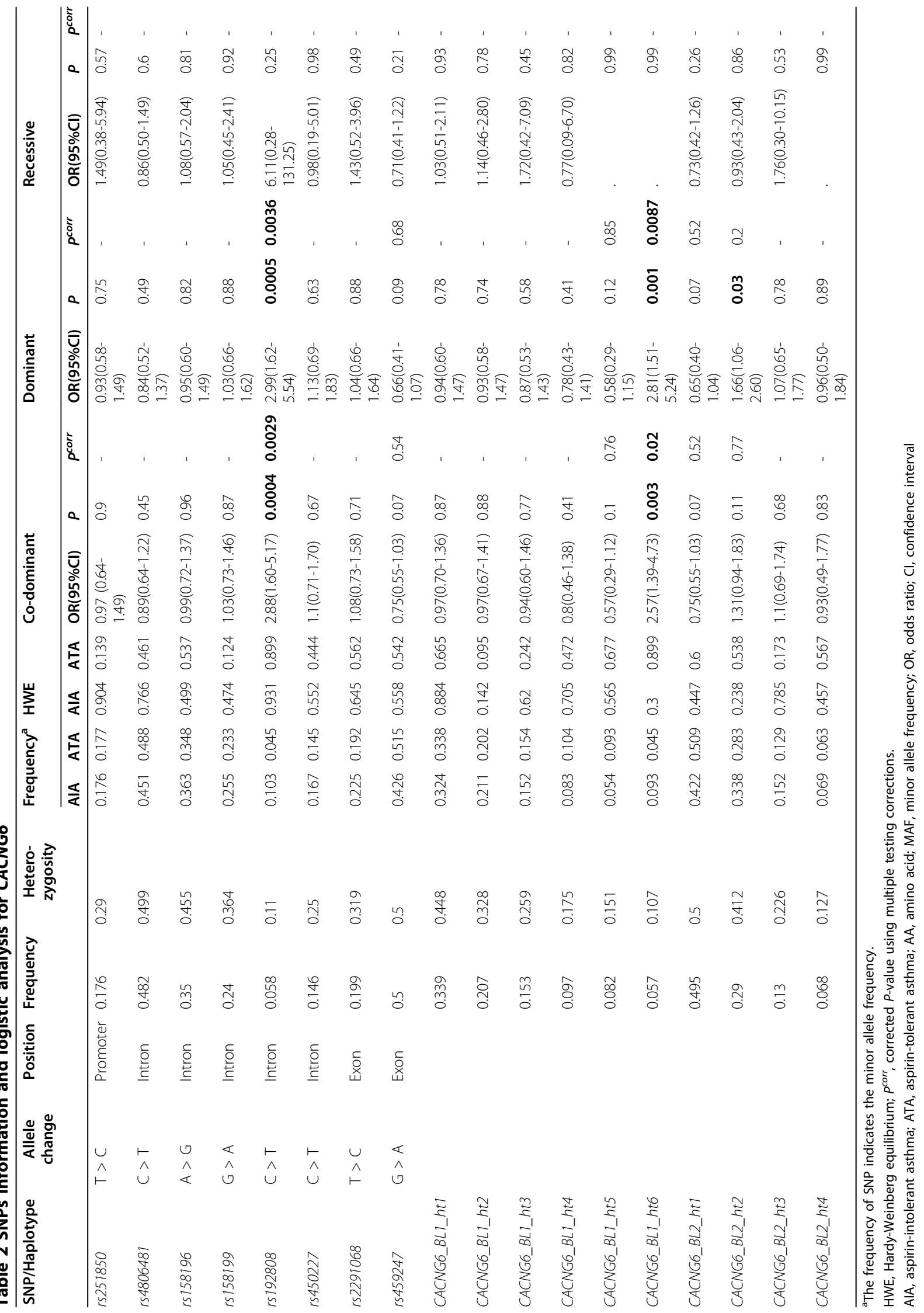


A. Map of $C A C N G 6$ (calcium channel, voltage-dependent, gamma subunit 6) on chromosome 19q13.4 (20kb) NM_145814.1

\begin{tabular}{|c|c|c|c|c|c|c|}
\hline \multirow[t]{6}{*}{$r^{2}=0.14$} & $r^{2}=0.09$ & $r^{2}=0.05$ & $r^{2}=0.01$ & $r^{2}=0.01$ & $r^{2}=0.004$ & $r^{2}=0.01$ \\
\hline & $r^{2}=0.04$ & $r^{2}=0.16$ & $r^{2}=0.06$ & $r^{2}=0.001$ & $r^{2}=0.00$ & $r^{2}=0.08$ \\
\hline & & $r^{2}=0.56$ & $r^{2}=0.03$ & $p^{2}=0.24$ & $r^{2}=0.10$ & $r^{2}=0.19$ \\
\hline & & & $r^{2}=0.01$ & $r^{2}=0.04$ & $r=0.03$ & $r^{2}=0.09$ \\
\hline & & & & $r^{2}=0.01$ & $r^{2}=0.01$ & $p^{2}=0.04$ \\
\hline & & & & & $r^{2}=0.52$ & $p^{2}=0.14$ \\
\hline
\end{tabular}

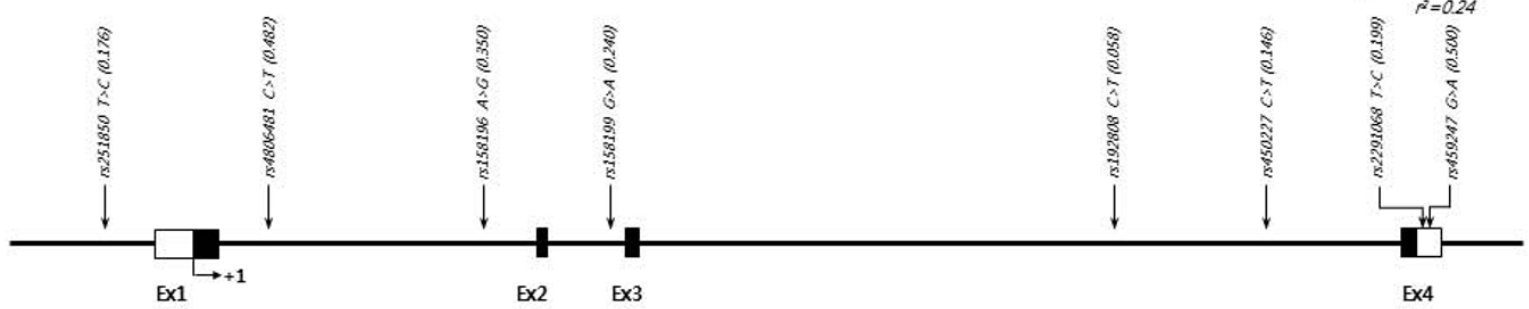

B. Haplotypes in CACNG6

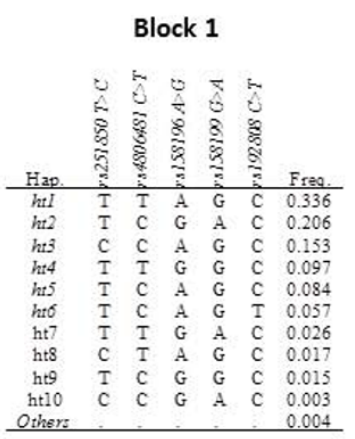

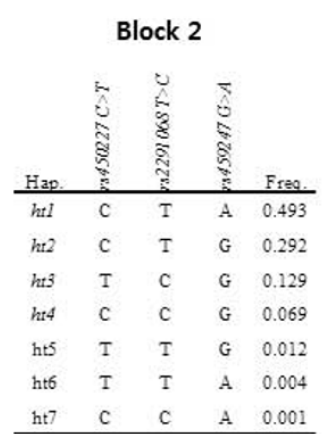

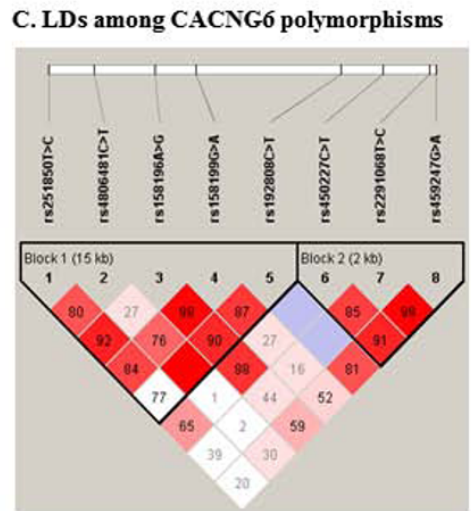

Figure 1 Gene maps and haplotypes of the CACNG6 gene. Schematic physical map, haplotypes and LD plot of CACNG6. (A) Polymorphisms identified in CACNG6. Coding exons are marked by shaded blocks and $3^{\prime}$-untranslated region (UTR) by white blocks. The LD coefficients $\left(r^{2}\right)$ are based on the genotypes of Korean samples. (B) Haplotypes of CACNG6 in the Korean population. Only those with frequencies over 0.05 are shown in Table 2 and 3. (C) LD coefficients $\left(\left|D^{\prime}\right|\right)$ among the selected SNPs based on the genotypes of whole study subjects in this study. (D) LD coefficients $\left(\left|D^{\prime}\right|\right)$ in AIA and ATA.

found that CACNG6 has three transcripts. Thus, to predict role of $r s 192808$, we performed GeneSplicer http://www. cbcb.umd.edu/software/GeneSplicer and EMBL-EBI splice site prediction tool http://www.ebi.ac.uk/asd-srv/wb.cgi? method $=2$ to know whether $r s 192808$ could be located in splicing association site [29]. However, results showed that the $r s 192808$ was not located in any splicing-related site such as donor site ( $5^{\prime}$ boundary), acceptor site ( $3^{\prime}$ boundary) and branch point. In addition, the functionality of other SNPs that were in LD with $r s 192808$ was analyzed. However, results from the Signal Scan program http://wwwbimas.cit.nih.gov/molbio/signal/ revealed that CACNG6 rs 251850 in promoter region was not a transcriptional element, and three intronic SNPs (rs4806481, rs158196, rs 158199) were not predicted as splicing-related sites.

A previous study has reported that some SNPs located deep in intronic region can create new transcription factor binding site [30]. Thus, we further performed TFSEARCH http://www.cbrc.jp/research/db/TFSEARCH.html using bioinformatical software to predict whether rs192808 can create transcription binding site. However, results showed that the location of $r s 192808$ is not in the transcription factor binding site. Even if these results suggest that $r s 192808$ may not affect the function of CACNG6, previous studies reported that sometimes allelic variants in intron can lead to the onset of human diseases due to alterations in the expression level of mRNA caused by creating potential aberrant splice sites [31,32]. Moreover, additional transcriptional isoforms of the CACNG6 gene have been discovered from the Mammalian Gene Collection (MGC) Program [33] as shown in the Ensembl Genome Browser http://www.ensembl.org/index.html (Additional files 3, Figure S1). Therefore, findings from our study on the significant association between rs 192808 and AIA needs to be replicated and/or further analyzed in order to understand its detailed function in the mechanism of AIA.

Since there is a possibility that CACNG6 SNPs are in LD with functional SNP(s) that is/are located in a different gene 
Table 3 Regression analysis between SNPs and haplotypes of CACNG6 in all subjects

\begin{tabular}{|c|c|c|c|c|c|c|c|}
\hline SNP or Haplotype & Position & $\mathrm{C} / \mathrm{C}$ & $\mathrm{C} / \mathrm{R}$ & $R / R$ & $\mathrm{~Pa}$ & $\mathrm{~Pb}$ & $P c$ \\
\hline$r s 251850$ & Promoter & $397(9.41 \pm 13.26)$ & $180(8.48 \pm 12.24)$ & $15(13.60 \pm 21.54)$ & 0.91 & 0.57 & 0.19 \\
\hline rs4806481 & Intron & $153(10.09 \pm 13.84)$ & $308(9.34 \pm 13.47)$ & $129(8.07 \pm 11.90)$ & 0.25 & 0.38 & 0.32 \\
\hline rs158196 & Intron & $251(9.19 \pm 13.69)$ & $265(8.81 \pm 12.70)$ & $76(10.85 \pm 13.47)$ & 0.75 & 0.79 & 0.29 \\
\hline rs158199 & Intron & $349(8.92 \pm 12.97)$ & $204(9.63 \pm 13.63)$ & $39(9.97 \pm 13.55)$ & 0.75 & 0.78 & 0.81 \\
\hline rs192808 & Intron & $529(8.81 \pm 12.99)$ & $60(12.35 \pm 13.94)$ & $3(21.67 \pm 28.36)$ & 0.006 & 0.01 & 0.06 \\
\hline CACNG6_BL1_ht1 & & $259(9.55 \pm 13.60)$ & $271(9.11 \pm 12.76)$ & $62(8.45 \pm 13.77)$ & 0.64 & 0.68 & 0.73 \\
\hline CACNG6_BL1_ht2 & & $382(9.19 \pm 13.48)$ & $178(9.05 \pm 12.56)$ & $32(10.72 \pm 13.99)$ & 0.98 & 0.82 & 0.59 \\
\hline CACNG6_BL1_ht3 & & $421(9.34 \pm 13.30)$ & $159(8.41 \pm 11.88)$ & $12(16.39 \pm 23.38)$ & 0.92 & 0.64 & 0.07 \\
\hline CACNG6_BL1_ht4 & & $481(9.40 \pm 13.60)$ & $104(8.67 \pm 11.80)$ & $7(6.14 \pm 2.97)$ & 0.5 & 0.56 & 0.57 \\
\hline CACNG6_BL1_ht5 & & $493(9.52 \pm 13.23)$ & $96(7.91 \pm 13.32)$ & $3(4.67 \pm 2.31)$ & 0.32 & 0.33 & 0.66 \\
\hline CACNG6_BL1_ht6 & & $530(8.83 \pm 12.99)$ & $60(12.90 \pm 14.91)$ & $2(5.50 \pm 6.36)$ & 0.03 & 0.02 & 0.69 \\
\hline rs450227 & Intron & $426(9.18 \pm 13.11)$ & $157(9.38 \pm 13.56)$ & $9(9.33 \pm 13.76)$ & 0.89 & 0.91 & 0.87 \\
\hline rs2291068 & Exon & $378(9.17 \pm 13.17)$ & $194(9.40 \pm 13.14)$ & $20(8.72 \pm 15.41)$ & 0.69 & 0.79 & 0.61 \\
\hline rs459247 & Exon & $150(11.12 \pm 15.52)$ & $290(8.74 \pm 11.28)$ & $152(8.32 \pm 14.07)$ & 0.12 & 0.06 & 0.48 \\
\hline CACNG6_BL2_ht1 & & $153(11.09 \pm 15.38)$ & $290(8.70 \pm 11.28)$ & $149(8.37 \pm 14.19)$ & 0.12 & 0.06 & 0.50 \\
\hline CACNG6_BL2_ht2 & & $301(8.17 \pm 12.70)$ & $240(10.55 \pm 13.87)$ & $51(9.34 \pm 12.78)$ & 0.09 & 0.03 & 0.97 \\
\hline CACNG6_BL2_ht3 & & $442(9.37 \pm 13.28)$ & $145(8.72 \pm 12.92)$ & $5(11.80 \pm 18.75)$ & 0.51 & 0.44 & 0.75 \\
\hline CACNG6_BL2_ht4 & & $515(9.12 \pm 13.08)$ & $76(9.99 \pm 14.29)$ & $1(8.00)$ & 0.86 & 0.83 & 0.78 \\
\hline
\end{tabular}

$\mathrm{C} / \mathrm{C}$, common allele/common allele; $\mathrm{C} / \mathrm{R}$, common allele/rare allele; $\mathrm{R} / \mathrm{R}$, rare allele/rare allele;

$P a, P$-values of co-dominant model; $P b$, dominant model; $P C$, recessive model.

in the nearby region, we further analyzed the LD near CACNG6 in Asian populations (Japanese and Chinese) from the International HapMap Project. However, the CACNG6 gene showed no LD with other nearby genes (Additional files 4, Figure S2). On the other hand, although minor allele frequencies of 8 SNPs validated in Korean asthmatics were similar to those of Asian populations (Chinese and Japanese), significant differences were detected from other populations especially in rs158196, rs1581199, rs 192808, rs450227, rs2291068, and rs459247 (Additional files 2, Table S2). When comparing LDs on 8 SNPs of CACNG6 among Korean asthmatic patients and other populations (Additional file 5, Figure S3), albeit non-asthmatic Korean population has not determined, it can be assumed that a different LD status of the asthmatics may affect AIA and/or asthmarelated phenotypes at least in a Korean population. Moreover, although replications and functional studies are needed, rs192808 and CACNG6_BL1_ht6 could be genetic markers of AIA susceptibility.

\section{Conclusions}

Although replication for the associations is required in an independent study cohort to validate our findings, this study showed that the genetic polymorphisms of CACNG6 might be associated with risk of AIA at least in a Korean population, providing a new link between voltage-dependent calcium channel and aspirin hypersensitivity in asthmatics. Further functional and replication studies elsewhere are needed to identify the roles of the polymorphisms of the gene.

\section{Additional material}

Additional file 1: Regression analysis between SNPs and haplotypes of CACNG6 in patients with AIA only. Results of regression analysis in AIA patients only

Additional file 2: Allele information in other population for CACNG6. Allele frequencies of CACNG6 polymorphisms among Korean and other populations

Additional file 3: Three isoforms of CACNG6. Three different types of the CACNG6 gene transcripts are found from the Ensembl Genome Browser http://www.ensembl.org/index.html.

Additional file 4: LD plot nearby CACNG6. LD blocks nearby CACNG6 based on Asian populations (Japanese and Chinese) show no LD between CACNG6 and near genes. (A) LD blocks of near genes and CACNG6. Data for LD map is obtained from the International HapMap project. (B) LD block of the CACNG6 gene.

Additional file 5: LDs of CACNG6 polymorphisms among populations. Comparison of LDs of CACNG6 between Korean asthmatics and other populations. (A) Korean asthmatics. (B) Korean AIA. (C) Korean ATA. (D) Caucasian. (E) Asians including Japanese and Chinese. (F) Africans

\section{List of Abbreviations}

AIA: aspirin-intolerant asthma; ATA: aspirin-tolerant asthma; CACNG6: calcium channel: voltage-dependent: gamma subunit 6; SNP: single nucleotide polymorphism; MAF: minor allele frequency; LD: linkage disequilibrium; OR: odds ratio; Cl: confidence interval

\section{Competing interests}

The authors declare that they have no competing interests.

\section{Authors' contributions}

JSL developed tables/figures, and drafted the manuscript. JK, JSB and CFP helped to interpret the data and to draft the manuscript. SU, JP, AJ, MK, ISC and CP recruited subjects. TJP and JYK, participated in preparation and quality control of samples, and data collection. BP and HSC performed the 
statistical analysis. CP and HDS coordinated all of this study and helped to draft the manuscript. All authors read and approved the final manuscript.

\section{Acknowledgements}

This work was supported by a grant from the Korea Health 21 R\&D Project (A010249); a grant number M1-0302-00-0073 from Korea Science and Engineering Foundation (KOSEF) funded by the Korea government (MEST) (No. 2009-0080157); an Intramural Research Grant of the Korea National Institute of Health (grant number 4800-4845-300-260-00); and a Priority Research Centers Program through the National Research Foundation of Korea (NRF) funded by the Ministry of Education, Science and Technology (2009-0093822). The DNA samples were generously provided by the Soonchunhyang University, Bucheon Hospital Biobank, a member of the National Biobank of Korea, supported by the Ministry of Health, Welfare and Family Affairs, Republic of Korea.

\section{Author details}

${ }^{1}$ Department of Life Science, Sogang University, Seoul 121-742, Republic of Korea. ${ }^{2}$ Department of Genetic Epidemiology, SNP Genetics, Inc., Seoul 153801, Republic of Korea. ${ }^{3}$ Division of Allergy and Respiratory Medicine,

Soonchunhyang University Seoul Hospital, Seoul 140-743, Republic of Korea. ${ }^{4}$ Genome Research Center for Allergy and Respiratory Disease,

Soonchunhyang University Bucheon Hospital, Bucheon 420-767, Republic of Korea. ${ }^{5}$ Division of Internal Medicine, Chungbuk National University, College of Medicine, Cheongju 361-711, Republic of Korea. 'Department of Allergy, Chonnam National University Medical School and Research Institute of Medical Sciences, Gwangju 501-757, Republic of Korea.

Received: 22 June 2010 Accepted: 23 September 2010

Published: 23 September 2010

\section{References}

1. Widal F, Abrami P, Lermoyez J: Anaphylaxie et idiosyncrasie. 1992 [Anaphylaxis and idiosyncrasy. 1992]. Allergy Proc 1993, 14(5):373-376, discussion 371-372.

2. Szczeklik A, Stevenson DD: Aspirin-induced asthma: advances in pathogenesis, diagnosis, and management. J Allergy Clin Immunol 2003, 111(5):913-921, quiz 922

3. Samter M, Beers RF Jr: Concerning the nature of intolerance to aspirin. Allergy 1967, 40(5):281-293.

4. Stevenson DD, Sanchez-Borges M, Szczeklik A: Classification of allergic and pseudoallergic reactions to drugs that inhibit cyclooxygenase enzymes. Ann Allergy Asthma Immunol 2001, 87(3):177-180.

5. Pawankar R: Nasal polyposis: an update: editorial review. Curr Opin Allergy Clin Immunol 2003, 3(1):1-6.

6. Funk CD: Prostaglandins and leukotrienes: advances in eicosanoid biology. Science 2001, 294(5548):1871-1875.

7. Chang WC, Nelson C, Parekh AB: Ca2+ influx through CRAC channels activates cytosolic phospholipase $A 2$, leukotriene $C 4$ secretion, and expression of c-fos through ERK-dependent and -independent pathways in mast cells. Faseb J 2006, 20(13):2381-2383.

8. Di Capite J, Shirley A, Nelson C, Bates G, Parekh AB: Intercellular Ca2+ wave propagation involving positive feedback between CRAC channels and cysteinyl leukotrienes. Faseb J 2009, 23(3):894-905.

9. Chang WC, Parekh AB: Close functional coupling between $\mathrm{Ca} 2+$ releaseactivated $\mathrm{Ca} 2+$ channels, arachidonic acid release, and leukotriene C4 secretion. J Biol Chem 2004, 279(29):29994-29999.

10. Chang WC, Di Capite J, Nelson C, Parekh AB: All-or-none activation of CRAC channels by agonist elicits graded responses in populations of mast cells. J Immunol 2007, 179(8):5255-5263.

11. Cocks TM, Moffatt JD: Protease-activated receptor-2 (PAR2) in the airways. Pulm Pharmacol Ther 2001, 14(3):183-191.

12. Morello S, Vellecco V, Roviezzo F, Maffia P, Cuzzocrea S, Cirino G, Cicala C: A protective role for proteinase activated receptor 2 in airways of lipopolysaccharide-treated rats. Biochem Pharmacol 2005, 71(1-2):223-230.

13. Sanderson MJ, Delmotte P, Bai Y, Perez-Zogbhi JF: Regulation of airway smooth muscle cell contractility by $\mathrm{Ca} 2+$ signaling and sensitivity. Proc Am Thorac Soc 2008, 5(1):23-31.

14. Brueggemann LI, Mani BK, Mackie AR, Cribbs LL, Byron KL: Novel Actions of Nonsteroidal Anti-Inflammatory Drugs on Vascular lon Channels:
Accounting for Cardiovascular Side Effects and Identifying New Therapeutic Applications. Mol Cell Pharmacol 2(1):15-19.

15. Glossmann H, Striessnig J, Ferry DR, Goll A, Moosburger K, Schirmer M: Interaction between calcium channel ligands and calcium channels. Circ Res 1987, 61(4 Pt 2):130-36.

16. Wang IM, Stepaniants S, Boie Y, Mortimer JR, Kennedy B, Elliott M, Hayashi S, Loy L, Coulter S, Cervino S, et al: Gene expression profiling in patients with chronic obstructive pulmonary disease and lung cancer. Am J Respir Crit Care Med 2008, 177(4):402-411.

17. Heguy A, Harvey BG, Leopold PL, Dolgalev I, Raman T, Crystal RG: Responses of the human airway epithelium transcriptome to in vivo injury. Physio/ Genomics 2007, 29(2):139-148.

18. Lin Z, Witschas K, Garcia T, Chen RS, Hansen JP, Sellers ZM, Kuzmenkina E, Herzig S, Best PM: A critical GxxxA motif in the gamma6 calcium channel subunit mediates its inhibitory effect on Cav3.1 calcium current. J Physiol 2008, 586(Pt 22):5349-5366

19. Li S, Blaschke M, Heubach JF, Wettwer E, Ravens U: Effects of azelastine on contractility, action potentials and L-type $\mathrm{Ca}(2+)$ current in guinea pig cardiac preparations. Eur J Pharmacol 2001, 418(1-2):7-14.

20. Wong CW, Luis S, Zeng I, Stewart RA: Eosinophilia and coronary artery vasospasm. Heart Lung Circ 2008, 17(6):488-496.

21. Akahoshi M, Obara K, Hirota T, Matsuda A, Hasegawa K, Takahashi N, Shimizu M, Nakashima K, Cheng L, Doi S, et al: Functional promoter polymorphism in the TBX21 gene associated with aspirin-induced asthma. Hum Genet 2005, 117(1):16-26.

22. Park HS: Early and late onset asthmatic responses following lysine-aspirin inhalation in aspirin-sensitive asthmatic patients. Clin Exp Allergy 1995, 25(1):38-40.

23. Hedrick PW: Gametic disequilibrium measures: proceed with caution. Genetics 1987, 117(2):331-341

24. Stephens M, Smith NJ, Donnelly P: A new statistical method for haplotype reconstruction from population data. Am J Hum Genet 2001, 68(4):978-989.

25. Tantisira KG, Drazen JM: Genetics and pharmacogenetics of the leukotriene pathway. J Allergy Clin Immunol 2009, 124(3):422-427.

26. Kim SH, Ye YM, Lee SK, Choi JH, Holloway JW, Park CS, Park HS: Association of TNF-alpha genetic polymorphism with HLA DPB1*0301. Clin Exp Allergy 2006, 36(10):1247-1253.

27. Hitomi Y, Ebisawa M, Tomikawa M, Imai T, Komata T, Hirota T, Harada M, Sakashita M, Suzuki $Y$, Shimojo N, et al: Associations of functional NLRP3 polymorphisms with susceptibility to food-induced anaphylaxis and aspirin-induced asthma. J Allergy Clin Immunol 2009, 124(4):779-785, e776.

28. Dahlen SE, Hedqvist P, Hammarstrom S, Samuelsson B: Leukotrienes are potent constrictors of human bronchi. Nature 1980, 288(5790):484-486.

29. Pertea $M$, Lin X, Salzberg SL: GeneSplicer: a new computational method for splice site prediction. Nucleic Acids Res 2001, 29(5):1185-1190.

30. Pagani F, Buratti E, Stuani C, Bendix R, Dork T, Baralle FE: A new type of mutation causes a splicing defect in ATM. Nat Genet 2002, 30(4):426-429.

31. Amsellem S, Briffaut $D$, Carrie A, Rabes JP, Girardet JP, Fredenrich A, Moulin P, Krempf M, Reznik Y, Vialettes B, et al: Intronic mutations outside of Alu-repeat-rich domains of the LDL receptor gene are a cause of familial hypercholesterolemia. Hum Genet 2002, 111(6):501-510.

32. King K, Flinter FA, Nihalani $V$, Green PM: Unusual deep intronic mutations in the COL4A5 gene cause $\times$ linked Alport syndrome. Hum Genet 2002, 111(6):548-554.

33. Strausberg RL, Feingold EA, Grouse LH, Derge JG, Klausner RD, Collins FS, Wagner L, Shenmen CM, Schuler GD, Altschul SF, et al: Generation and initial analysis of more than 15,000 full-length human and mouse CDNA sequences. Proc Natl Acad Sci USA 2002, 99(26):16899-16903.

\section{Pre-publication history}

The pre-publication history for this paper can be accessed here: http://www.biomedcentral.com/1471-2350/11/138/prepub

\section{doi:10.1186/1471-2350-11-138}

Cite this article as: Lee et al:: Association of CACNG6 polymorphisms with aspirin-intolerance asthmatics in a Korean population. BMC Medical Genetics 2010 11:138. 